\title{
Lidar research activities in Potenza, Southern Italy
}

\author{
Paolo Di Girolamo $\left({ }^{1}\right)$, Aldo Amodeo $\left({ }^{2}\right)$, \\ Marco Pandolfi $\left({ }^{2}\right)$, Gelsomina Pappalardo $\left({ }^{2}\right)$ and Vincenzo Cuomo $\left({ }^{1}\right)\left({ }^{2}\right)$ \\ (1) Dipartimento di Ingegneria e Fisica dell'Ambiente (DIFA), Università della Basilicata, Potenza, Italy \\ $\left(^{2}\right)$ Istituto di Metodologie per l'Analisi Ambientale (IMAA), CNR, Tito Scalo (PZ), Italy
}

\begin{abstract}
The lidar group in Potenza has almost 10 years' experience in the field of lidar research. Important results have been accomplished both in aerosol and water vapour research. The lidar system in Potenza has acquired different configurations during the years, always preserving the capability to accomplish two wavelength aerosol and water vapour Raman measurements. An important up-grade was the introduction of a water vapour DIAL channel in 1997. The lidar system in Potenza has been involved in several aerosol and water vapour measurement campaigns: stratospheric aerosol measurement campaign (1994-1995); LITE correlative measurement campaign (September 1994); Water Vapour Intensive Observation Period (January-February 1997); EARLINET project (since February 2000). A second lidar system, primarily dedicated to water vapour Raman measurements, is under development and starting January 2002 will go through an intensive observation period dedicated to the validation of sensors on-board ENVISAT (principally GOMOS, MIPAS and SCIAMACHY). This paper summarises some of the major results accomplished, as well as expected results from the forthcoming campaigns.
\end{abstract}

Key words lidar - water vapour - aerosol

\section{Introduction}

Atmospheric water vapour and aerosols play a crucial role in the Earth climate, being a key element in the global radiation budget, in atmospheric circulation, as well as in the microphysical processes leading to cloud formation and development. The role of atmospheric water vapour and aerosols in the climate system is only partially understood at present, as a result of their highly variable time and space distribution on the global scale. Global coverage

Mailing address: Dr. Paolo Di Girolamo, Dipartimento di Ingegneria e Fisica dell' Ambiente (DIFA), Università della Basilicata, C.da Macchia Romana, 85100 Potenza, Italy; e-mail: digirolamo@unibas.it measurements of aerosol and water vapour with high accuracy, and high spatial and temporal resolution are needed throughout the troposphere up to the lower stratosphere.

In spite of the recent improvements in their performances, passive remote sensors from space provide global coverage of aerosol and $\mathrm{H}_{2} \mathrm{O}$ distribution with accuracy and a vertical resolution still insufficient for climate studies, primarily in the middle and upper troposphere.

The lidar system based both on the Raman and DIAL techniques can provide accurate measurements of atmospheric water vapour, as well as aerosols, with high space and time resolution. Ground-based and airborne lidar systems operational spread around the globe, as well as the Shuttle-based experiment LITE, have demonstrated the technological maturity of the lidar technique for operational space-borne application.

In this paper we review the major results accomplished by one of these systems, namely 
the lidar system in Potenza. We intend to illustrate the performed measurement campaigns in terms of aerosol and water vapour measurements. The EARLINET project, mentioned in the abstract, is not included in the present work since a specific paper within this special issue is devoted to this effort.

\section{Instrumentation}

\subsection{The aerosol/water vapour system}

The lidar system in Potenza is located within the scientific laboratories of IMAA $\left(40^{\circ} 50^{\prime} \mathrm{N}\right.$, $14^{\circ} 10^{\prime} \mathrm{E}, 820 \mathrm{~m}$ a.s.l.) and was developed in co-operation with the Physics Department of the «Federico II» University of Naples. The system has been operated throughout the years by personnel from Instituto di Metodologie per l'Analisi Ambientale (IMAA) - Consiglio Nazionale delle Ricerche and University of Basilicata. It started to take measurements in July 1993. Figure 1 shows the block diagram of the system.

The Potenza lidar system was originally developed around a Nd:YAG laser source, including second and third harmonic generation crystals (532 and $355 \mathrm{~nm}$ ) and providing pulses at a repetition rate up to $20 \mathrm{~Hz}$. More recently, a second laser source was added, namely a dye laser, tunable within a spectral region of major absorption for water vapour $(690-730 \mathrm{~nm})$ (Ambrico et al., 1997; Di Girolamo et al., 1999). The dye laser source is pumped by a portion of the $532 \mathrm{~nm}$ beam.

As the laser pulses propagate through the atmosphere, part of their energy is backscattered to the instrument by particles - typically aerosols or hydrometeors - and by the molecules of the gas species comprising the atmospheric mixture. Laser beams at $355 \mathrm{~nm}$ and $532 \mathrm{~nm}$ are used to monitor aerosol and clouds. The $355 \mathrm{~nm}$ laser beam is also used to stimulate vibrational Raman scattering by water vapour and nitrogen molecules, at 407.5 and $386.6 \mathrm{~nm}$, respectively.

The dye laser is exploited for the application of the DIAL technique to water vapour monitoring. This laser source is able to transmit two laser beams at two different wavelengths ( $\lambda_{\text {on }}$ and $\left.\lambda_{\text {off }}\right)$, the first one falling on a water vapour absorption line center and the second falling on the line shoulder. For the measurements illustrated in the present paper, $\lambda_{\text {on }}$ is set to 723.59 , while $\lambda_{\text {off }}$ is $723.71 \mathrm{~nm}$.

The receiver consists of a vertically pointing cassegrainian telescope ( $0.5 \mathrm{~m}$ diameter primary mirror, $5 \mathrm{~m}$ combined focal length). The presence of a series of dichroic mirrors split the collected radiation into four portions $(355 \mathrm{~nm}$, $386 \mathrm{~nm}, 407 \mathrm{~nm}, 532 \mathrm{~nm}$ ). Interference filters or single/double grating monochromator are used as spectral selection devices. The introduction of a fused-silica beamsplitter transmits $50 \%$ of the collected radiation into the old section of the receiver, and deflects the remaining $50 \%$ into the DIAL channel. This particular partitioning is considered in order to obtain comparable performances for the Raman and DIAL techniques for the reported water vapour measurements. Detection is accomplished by means of photomultipliers. Detected signals are sampled by means of both analog-to-digital conversion and photon counting.

Water vapour DIAL measurements were supported by a photoacoustic apparatus, used to perform high-resolution measurements of the water vapour absorption spectrum and to check the dye laser tuning on the selected absorption line during the DIAL measurement. The photoacoustic experiment was developed around a vacuum cell equipped with fused silica windows; distilled water is contained in a small tanker inside the cell. Pressure variations inside the cell, resulting from water vapour absorption, are detected by a condenser microphone, whose signals are amplified and processed by an analog integrator and acquired by means of a digital oscilloscope.

\subsection{Cal/val lidar system}

A second lidar system, primarily dedicated to water vapour Raman measurements, is under development at IMAA. This system will mainly be used to validate ENVISAT data products. This system is based on a Nd:YAG laser equipped with third harmonic generation crystals (repetition rate up to $100 \mathrm{~Hz}$ ). The third 


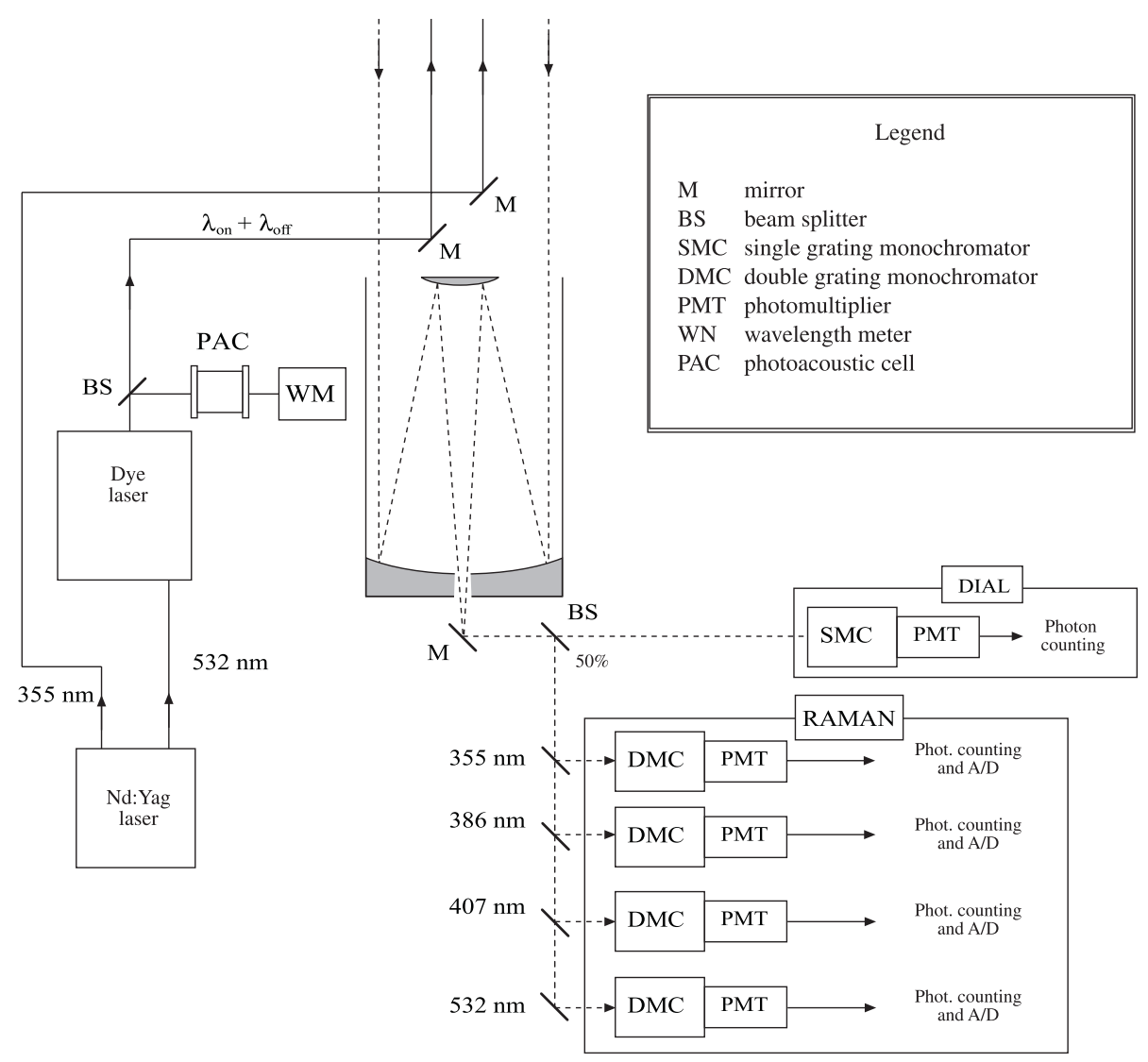

Fig. 1. Block diagram of the aerosol/water vapour lidar system.

harmonic is transmitted into the atmosphere in a coaxial mode. The receiver is developed around a vertically pointing telescope in Cassegrain configuration with a $0.5 \mathrm{~m}$ diameter primary mirror and a combined focal length of $5 \mathrm{~m}$. The collected radiation is split into three channels by means of dichroic mirrors. Interferential filters are used to select the elastic backscattered radiation at $355 \mathrm{~nm}$, the N2 Raman shifted signal at $386.6 \mathrm{~nm}$ and the water vapour Raman shifted signal at $407.5 \mathrm{~nm}$. Each wavelength is then split into 2 different channels for both low and high range signals. Photomultiplier tubes are used as detectors. Low range signals are sampled in analog mode by means of a digital oscilloscope (500 MHz). High range signals are acquired in photon counting mode using a Multi Channel Scaler (MCS) board with a dwell time of $100 \mathrm{~ns}$. Lidar measurements will be performed simultaneously with radiosonde launches to calibrate lidar measurements of water vapour.

\section{Main results}

\subsection{Stratospheric aerosol measurement campaign (1994-1995)}

Lidar measurements of stratospheric aerosol were made in Potenza on a routine basis in 
the period 1994-1995 (Di Girolamo et al., 1995, 1996). This measurement campaign is the continuation of the one started in Naples in September 1991. Measurements cover the history of the aerosol cloud produced by the eruption of Mount Pinatubo (June 1991, Philippines). The eruption of Mount Pinatubo in June 1991 determined a large increase in the stratospheric aerosol load on a global scale. This eruption is considered to be the most important of the century in terms of both volcanic material injected into the stratosphere and effects on the global climate (Zhao et al., 1995). Apart from the Pinatubo eruption, the period 1991-1995 was characterized by minor volcanic eruptions (Hudson, etc.) which produced negligible effects in the stratosphere on the global scale. This allowed aerosol sensors to observe the restoration of pre-volcanic constant background conditions and thus allowed to clearly distinguish and isolate the effects on a single eruption event.

Figure 2 shows the evolution with time of the stratospheric aerosol load. Data are expressed in terms of aerosol backscattering coefficient at 351
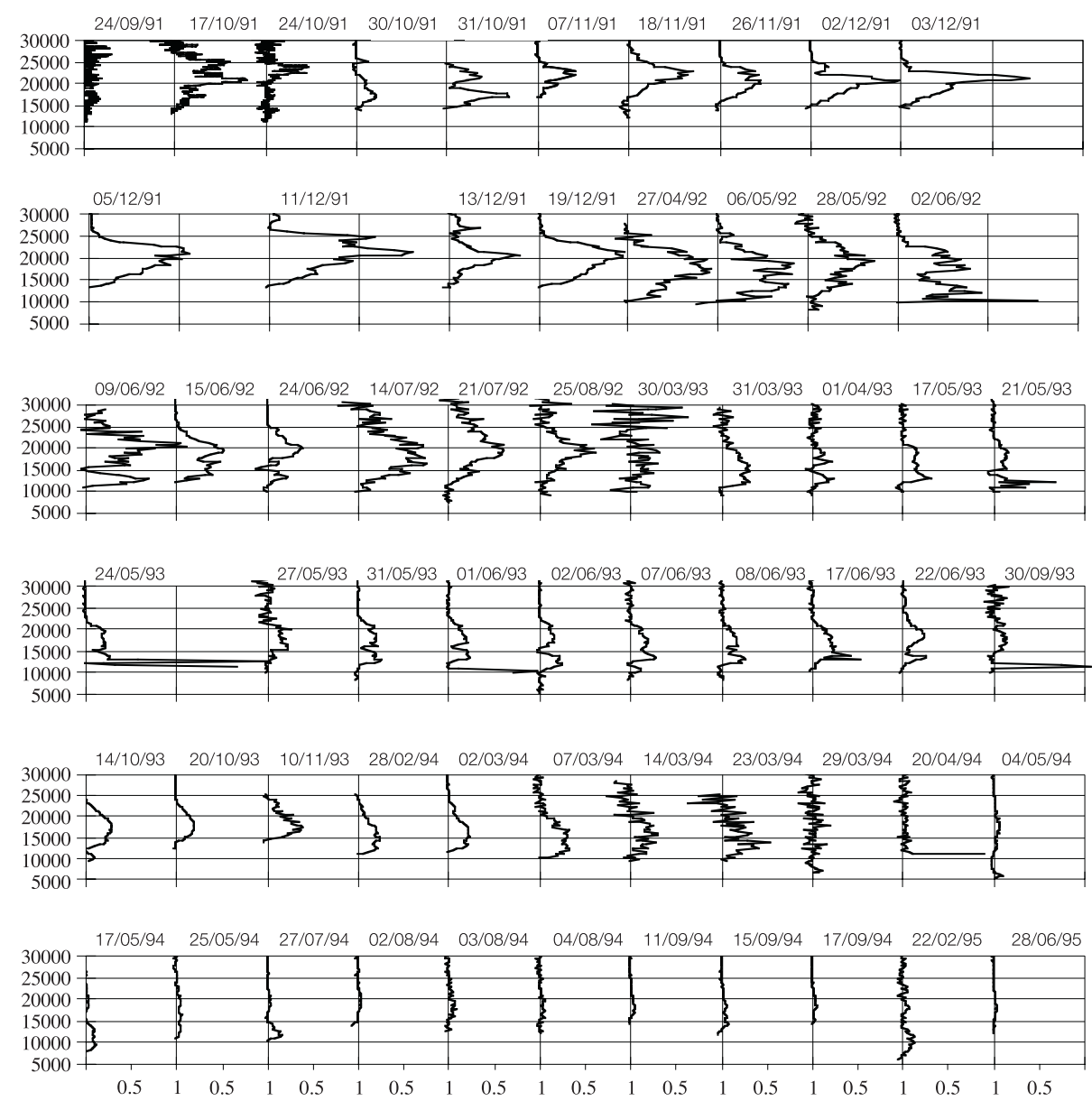

Fig. 2. Vertical profiles of the aerosol backscattering coefficient at 351 and $355 \mathrm{~nm}$ for 61 selected nights of measurements in the period September 1991 - June 1995. Data are expressed in units of $10^{-6} \mathrm{~m}^{-1} \mathrm{sr}^{-1}$. 
and $355 \mathrm{~nm}$. A large increase in aerosol load is observed starting September 1991, but maximum load is observed in December 1991 and decay started immediately afterwards.

Integrated Backscattering (IB) is obtained by integrating the aerosol backscattering coefficient throughout the stratosphere. IB (fig. 3) reached its maximum value in December 1991, displaying a subsequent decay with an e-folding times of $237 \pm 25$ days. Figure 4 shows the evolution with time of the aerosol optical thickness $\tau_{A}$. $\tau_{A}$ reached its maximum in December 1991, then decaying with an e-folding times of $250 \pm$ 111 days. $\tau_{A}$ is obtained from the elastic signal assuming an aerosol-free region above an below the stratospheric aerosol layer (Di Girolamo et al., 1996). While an aerosol free region is always present above the stratospheric aerosol layer, cirrus and upper tropospheric clouds can sometime prevent from locating an aerosol-free region below the stratospheric layer. Therefore, the number of data points for $\tau_{A}$ in fig. 4 is smaller than the number of data points of IB in fig. 3. Furthermore, the considered approach is particularly efficient for values of $\tau_{A}$ in excess of 0.3. Thus, in the second half of the data record (period 750-1500 days after eruption), when values of $\tau_{A}$ are often smaller than 0.3 , the number of data points in fig. 4 with respect to fig. 3 is further reduced.

Approximately 20 Mtons of $\mathrm{SO}_{2}$ were injected into the lower stratosphere within the first few days after the eruption (Bluth et al., 1992), and by late July 1991 the resulting aerosol cloud was diffused on a global scale (McCormick and Veiga,1992). The presence of a massive long-lasting stratospheric aerosol layer leads to an in-crease in the planetary albedo, resulting in a lower energy flux at surface level, as well as in an absorption enhancement within the aerosol layer. The latter leads to a temperature increase in the lower stratosphere, together with an enhancement of diffuse sky radiation. Effects of stratospheric aerosols of volcanic origin on the global radiation budget are dependent on the amount, height and lifetime of the material injected (Pollack et al., 1976). Observed values of the aerosol optical thickness (fig. 4) are consistent with a local increase in stratospheric temperature of 4-6 K.

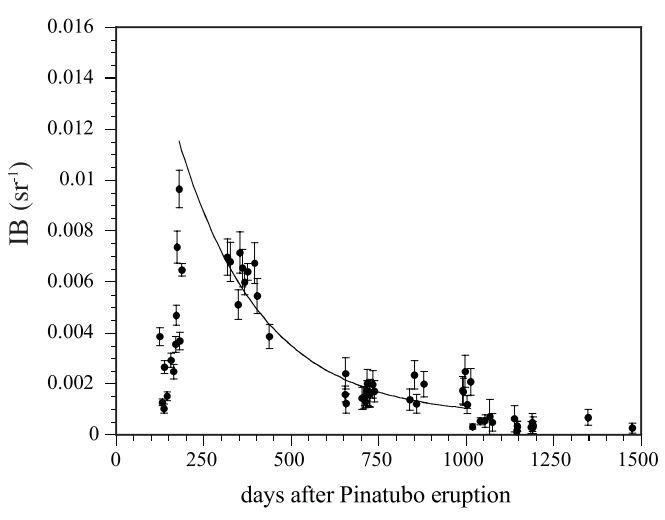

Fig. 3. Time evolution of integrated backscattering IB for the period 1991-1995. Also reported is the exponentially decreasing function fitted to the data.

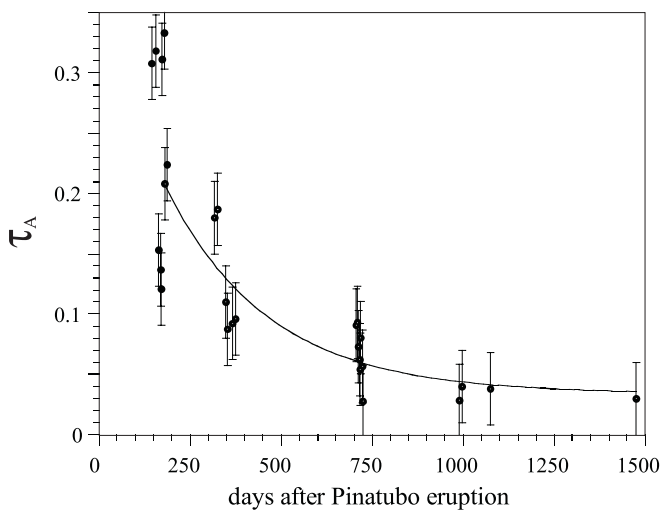

Fig. 4. Time evolution of the aerosol optical thickness $\tau_{A}$ for the period 1991-1995. Also reported is the exponentially decreasing function fitted to the data.

Figure 5 shows the evolution with time of the aerosol layer center of mass height, together with theoretical descent curves for different particle sizes as obtained from Kasten (1968). Theoretical data account just for sedimentation of aerosol particles, while vertical eddy diffusion is not considered. The figure clearly shows that data in the period 400-1000 days following the eruption follow the $0.3 \mu \mathrm{m}$ particle size curve, while data in the period 1000-1500 days after the eruption follow the $0.1 \mu \mathrm{m}$ particle curve. 


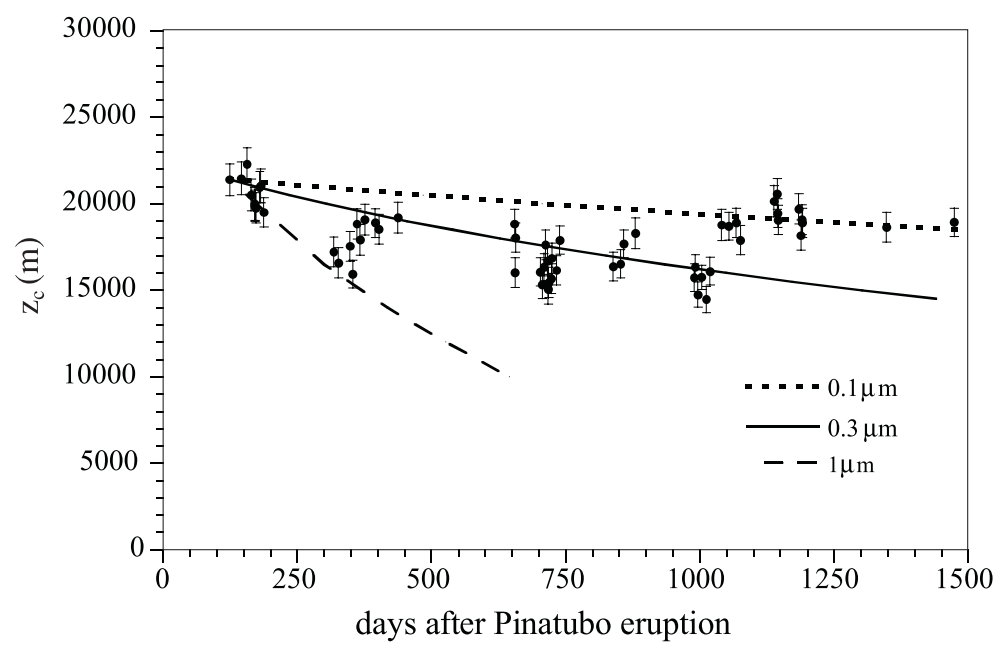

Fig. 5. Time evolution of $z_{c}$ for the period 1991-1995. Also reported are the theoretical descent curves for $1 \mu \mathrm{m}$, $0.3 \mu \mathrm{m}$ and $0.1 \mu \mathrm{m}$ diameter particles.

\subsection{LITE correlative measurement campain (September 1994)}

LITE (LIdar in space Technology Experiment), flying on the Space Shuttle in the period 10-19 September 1994, represented the first attempt to perform global coverage lidar measurements from space (McCormick et al., 1993). The lidar system in Potenza was involved together with another 60 lidar ground stations in the validation of LITE measurements. Thus, in conjunction with the LITE mission an intensive lidar measurement campaign was carried out in Potenza.

Because of the relative distance between LITE ground tracks and the validation station for all passes, measurements carried out in Potenza were primarily aimed to the validation of LITE stratospheric measurements (Cuomo et al., 1997,1998; Pappalardo et al., 1997). LITE accomplished 6 overpasses within $1500 \mathrm{~km}$ from Potenza. The minimum distance between LITE ground track and Potenza was $600 \mathrm{~km}$. Figure $6 \mathrm{a}, \mathrm{b}$ shows the comparison between simultaneous LITE and Potenza lidar measurements at both 355 (fig. 6a) and $532 \mathrm{~nm}$ (fig. 6b), in coincidence with Shuttle orbit 33. Data are expressed in terms of the aerosol backscattering ratio $R_{A}(z)$. Each

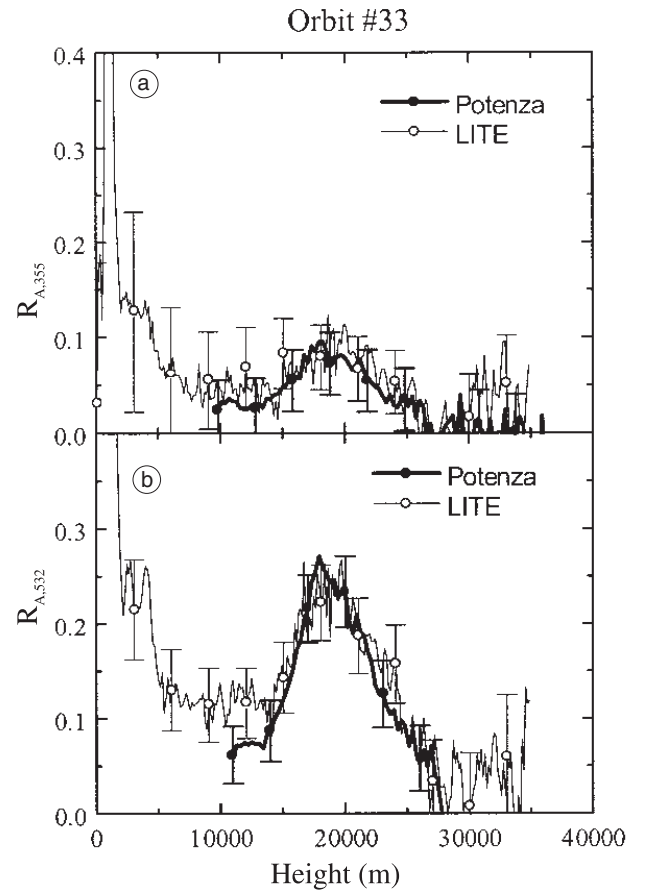

Fig. 6. Simultaneous LITE (thin line) and Potenza (solid line) lidar measurements of the aerosol scattering ratio at $355 \mathrm{~nm}$ (a) and $532 \mathrm{~nm}$ (b) for orbit 33. Data area reported with their error bars 


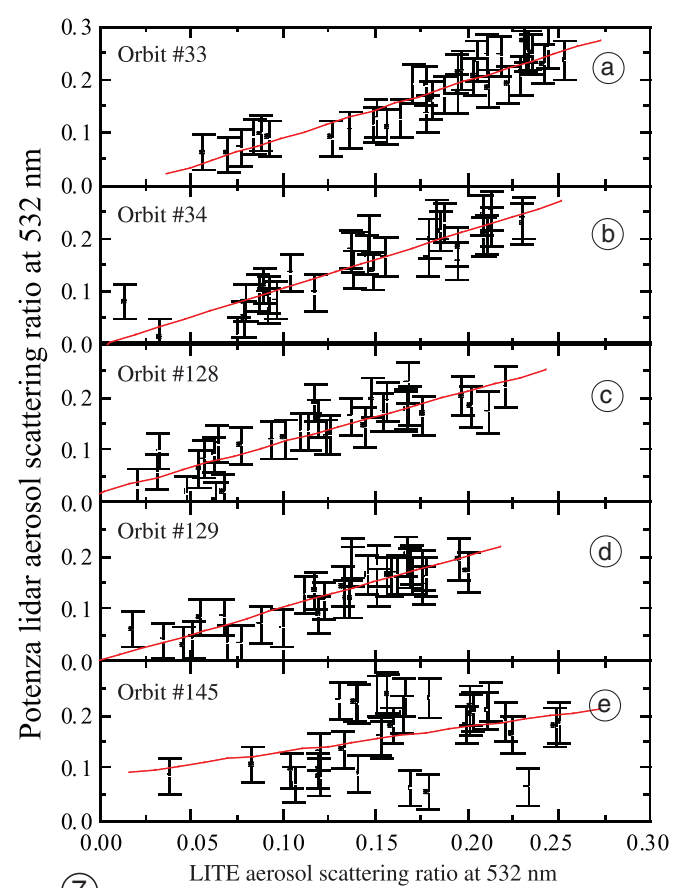

(7)

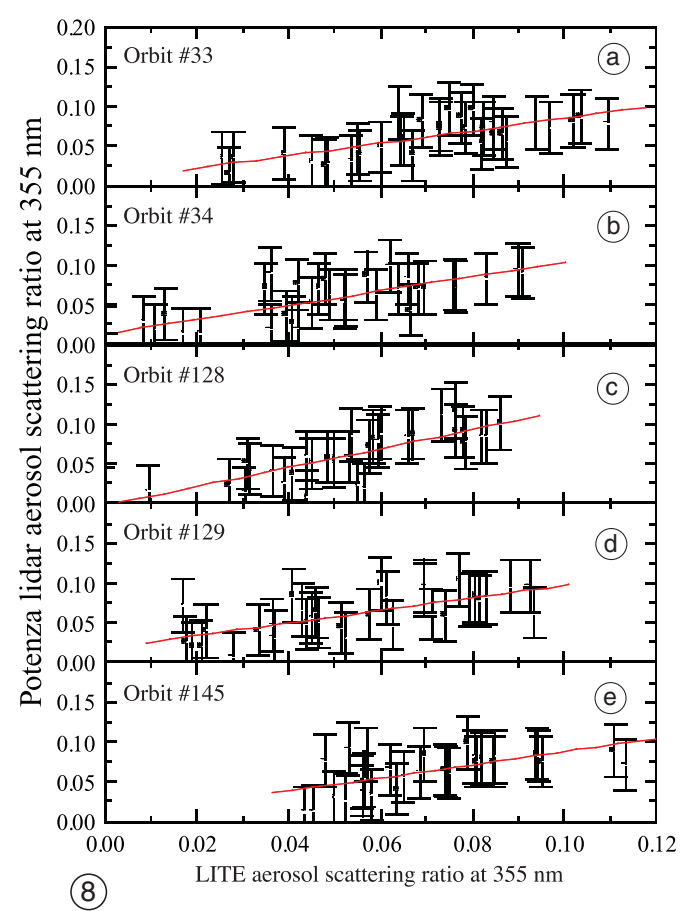

(8)

Fig. 7a-e. $R_{4532}^{\mathrm{LITE}}(z)$ versus $R_{A 532}^{\mathrm{Pz}}(z)$ for all data points within the stratospheric aerosol layer: a) orbit 33; b) orbit 34 ; c) orbit 128 ; d) orbit 129 and e) orbit 145 . Values of $R_{A, 532}^{\mathrm{LITE}}(z)$ are reported together with their error bars. Also reported is the best fit linear function.

Fig. 8a-e. $R_{A, 355}^{\mathrm{LTE}}(z)$ versus $R_{A, 35}^{\mathrm{Pz}}(z)$ for all data points within the stratospheric aerosol: a) orbit 33; b) orbit 34; c) orbit 128; d) orbit 129 and e) orbit 145, together with the best fit linear function.

LITE profile is averaged over 300 laser shots, with a vertical resolution of $150 \mathrm{~m}$, while each Potenza lidar profile is averaged over $90 \mathrm{~min}$, with a vertical resolution of $300 \mathrm{~m}$. Data are reported with their error bars.

Assuming that data at different altitudes are uncorrelated for both Potenza lidar and LITE, it is possible to make a statistical analysis of the two data sets to quantify the degree of agreement between the LITE and Potenza lidar measurements. Potenza lidar measurements of $R_{A, 532}(z)\left(R_{A, 532}^{\mathrm{Pz}}(z)\right)$ have been plotted as a function of corresponding LITE data $\left(R_{A, 532}^{\mathrm{LITE}}(z)\right)$; that is, for any given $z$ within the stratospheric aerosol layer, points of coordinates $\left(R_{A, 532}^{\mathrm{LTTE}}(z), R_{A, 532}^{\mathrm{Pz}}(z)\right)$ were considered (fig. 7a-e). Since LITE data have a vertical resolution of $150 \mathrm{~m}$, while Potenza lidar measurements have a resolution of $300 \mathrm{~m}$, the former data have been smoothed down to a resolution of $300 \mathrm{~m}$. This reduces the number of data points within the stratospheric aerosol layer from 70 to 35 ; however, the number is large enough to guarantee the applicability of the fitting procedure. Points reported in the plot are those corresponding to values of $z$ in the range 16-26 $\mathrm{km}$. Values of $R_{A, 532}^{\mathrm{Pz}}(z)$ are reported together with their error bars. The points in the plot have been least square fit by the linear function

$$
R_{A, 532}^{\mathrm{Pz}}(z)=m R_{A, 532}^{\mathrm{LITE}}(z)+c .
$$


Even if both $R_{A, 532}^{\mathrm{Pz}}(z)$ and $R_{A, 532}^{\mathrm{LITE}}(z)$ are affected by a non negligible uncertainty, only the error on $R_{\mathrm{A} 532}^{\mathrm{Pz}}(z)$ was considered in the fitting procedure. Fitting lines are presented in fig. 7a-e. The correlation coefficient of the fit, $R$, equal to 1 in case of perfect correlation, quantifies the degree of correlation between LITE and Potenza lidar data. If data analysis is properly performed, values of the fitting parameter $c$ are expected to be equal to 0 . The parameter $m$ gets the significance of a normalization coefficient to calibrate data: $m>1$ implies $R_{A, 532}^{\text {LTE }}(z)$ is underestimating $R_{A, 532}^{\mathrm{Pz}}(z)$ (assumed as truth for validation), while $m<1$ implies $R_{A, 532}^{\text {LITE }}(z)$ is overestimating $R_{A, 532}^{\mathrm{Pz}}(z)$. The fitting procedure was applied to data at both 532 (fig. 7a-e) and 355 nm (fig. 8a-e). Results can be summerized as follows. At $532 \mathrm{~nm}$, values of $R$ are in the range $0.88-0.93$ (average value of $0.90 \pm 0.02$ ). Such high values of $R$ imply a very good correlation between LITE and Potenza lidar data in terms of $R_{A, 532}(z)$. Values of $m$ are in the range 0.96-1.08, with an average value of $1.02 \pm 0.07$. At $355 \mathrm{~nm}$, values of $R$ are in the range 0.72-0.81 (average value of $0.77 \pm 0.04$ ) and values of $m$ are in the range 0.79-1.19 (average value of $0.92 \pm 0.19$ ). This latter result implies that LITE measurements at $355 \mathrm{~nm}$ are slightly underestimating Potenza lidar measurements.

\subsection{Water vapour intensive observation period (January-February 1997)}

Water vapour measurements in the troposphere up to approximately $10 \mathrm{~km}$ a.s.l. have been performed by means of a ground-based lidar system through the simultaneous application of the Raman and DIAL techniques.

Water vapour absorption lines were carefully selected to minimise DIAL measurement uncertainty. In particular, $\lambda_{\text {on }}$ was selected to limit interferences by molecular species different from the investigated one, to minimise the temperature variability of the water vapour absorption line strength and to meet the requirements in terms of optimal optical depth (Ambrico et al., 2000). The dependence on temperature and pressure of the water vapour absorption cross-section, as well as laser line alteration due to water vapour absorption and the effects of the limited laser spectral purity, have been carefully accounted for in the estimate of the altitude variability of the effective absorption cross-section, with particular attention to boundary layer measurements. Selected values for $\lambda_{\text {on }}$ and $\lambda_{\text {off }}$ are 723.59 and $723.71 \mathrm{~nm}$, respectively.

In early 1997 (20 January-20 February), an intensive observation period was devoted to the simultaneous application of the Raman and DIAL techniques for water vapour measurements. As an example of the performed measurements, fig. 9 shows the Raman measurement of the $n_{\mathrm{H}_{2} \mathrm{O}}(z)$ vertical profile carried out on February 18, 1997 (22:20-00:20 GMT). The figure also shows the simultaneous humidity profile measured by the DIAL technique (22:20-00:20 GMT) and by a radiosonde launched at 22:55 GMT. Measurements are expressed in terms of specific humidity $(\mathrm{g} / \mathrm{kg})$ and are reported with their error bars. Raman measurements extend up to approximately $9 \mathrm{~km}$, while DIAL measurements

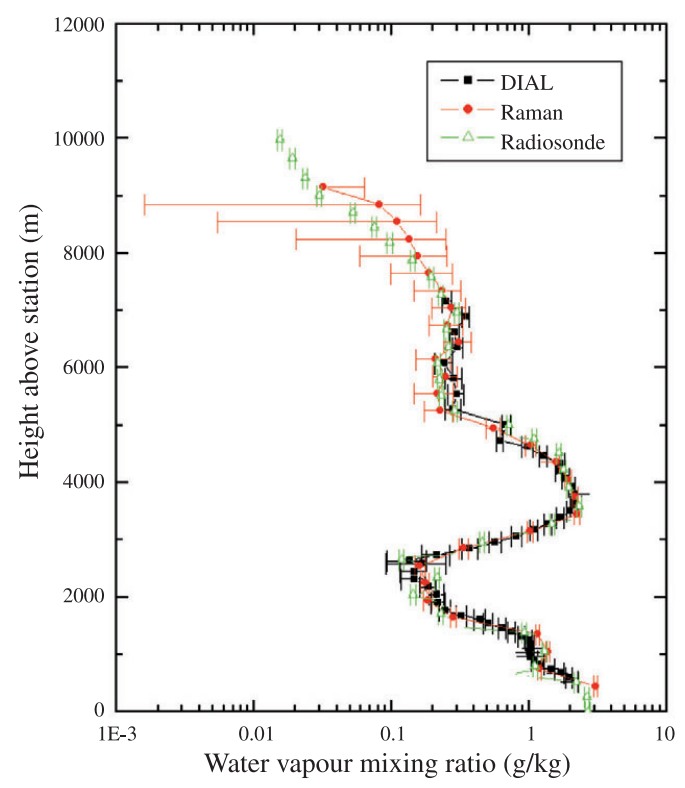

Fig. 9. Vertical profiles of $n_{\mathrm{H}_{2} \mathrm{O}}(z)$ for February 18, 1997: DIAL measurement (22:20-00:20 GMT, black squares), Raman measurement (22:20-00:20 GMT, red circles) and radiosonde profile (launch at 22:55, green triangles). 
display large error values above $7 \mathrm{~km}$ and have not been reported above this height.

The calibration coefficient for Raman data is obtained on the basis of an extended comparison between Raman measurements and simultaneous and co-located radiosonde measurements, the accuracy of Raman measurement being strictly related to the reliability of radiosonde humidity measurements used for the calibration. Since the DIAL technique is self-calibrating, water vapour density measurements can be performed in the troposphere by the DIAL technique with an uncertainty which is primarily limited by the DIAL signal statistical error.

Raman, DIAL and radiosonde measurements appear to be in good agreement up to approximately $8 \mathrm{~km}$ AGL, with a relative deviation which does not exceed $20 \%$ up to $7 \mathrm{~km}$ (fig. 9).

In the same period, measurements were also performed to characterize the boundary layer structure (Di Girolamo et al., 1999). Aerosols and moisture tend to be trapped within the PBL and can be used as tracers for the study of the boundary layer vertical structure and time variability (Pappalardo et al., 2001). Figure 10 shows the comparison between simultaneous lidar and radiosonde data for 11 February; lidar data are expressed in terms of aerosol backscattering coefficient at $723 \mathrm{~nm}$, while radiosonde data are expressed in terms of potential temperature and relative humidity. The potential temperature profile appears to be anti-correlated with both the relative humidity and the aerosol backscattering, the aerosol layer top being associated with a maximum in potential temperature and a minimum in relative humidity.

\subsection{Forthcoming measurements}

The lidar system described in Section 2.2 will go through an intensive observation period (starting January 2002) dedicated to the validation of the sensors on-board ENVISAT, primarily AATSR, GOMOS, MIPAS, MERIS and SCIAMACHY. AATSR will provide, among others, measurements of cloud top height. The lidar system will be used to validate these

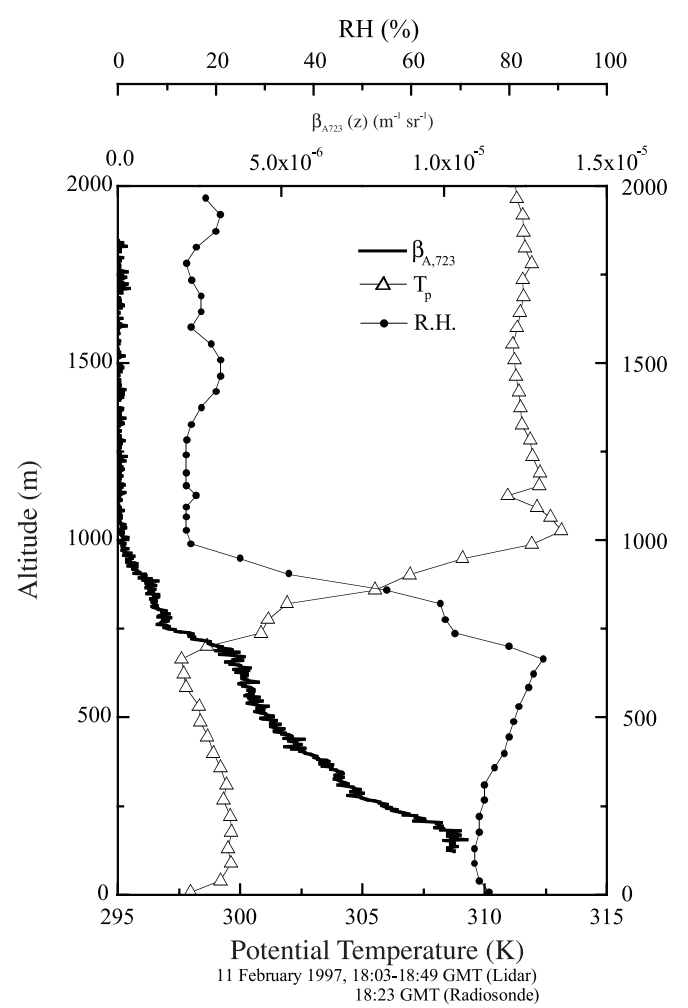

Fig. 10. Vertical profiles of aerosol backscattering coefficient at $723 \mathrm{~nm}$ for 11 February 1997 (bold line) and simultaneous radiosonde profiles of potential temperature (open up triangles) and relative humidity (solid dots)

measurements, primarily in the vertical interval $0.5-20 \mathrm{~km}$. MIPAS will provide measurements of several atmospheric constituents in the height region $8-53 \mathrm{~km}$. The lidar system will be used to validate MIPAS measurements of the water vapour profile in the vertical region $8-10 \mathrm{~km}$. Validation above this height (up to approximately $20 \mathrm{~km}$ ) will be provided by radiosondes. GOMOS measurements of stratospheric aerosol will be validated by lidar system up to approximately $20 \mathrm{~km}$. The system will also be used to validate MERIS and SCIAMACHY measurements of aerosol optical thickness. SCIAMACHY will also provide, among others, measurements of precipitable water to be validated by lidar. 
Table I. ENVISAT validation: list of validated and validating sensors.

CALVAL instrument

Geophysical parameter

CALVAL Vertical interval

CALVAL instrument

Geophysical parameter

CALVAL Vertical interval

CALVAL instrument

Geophysical parameter

CALVAL Vertical interval

CALVAL instrument

Geophysical parameter

CALVAL Vertical interval

Geophysical parameter

CALVAL Vertical interval

Geophysical parameter

CALVAL Vertical interval

CALVAL instrument

Geophysical parameter

CALVAL Vertical interval

CALVAL instrument

Geophysical parameter

CALVAL Vertical interval

Geophysical parameter

CALVAL Vertical interval

CALVAL instrument

Geophysical parameter

CALVAL Vertical interval

CALVAL instrument

Geophysical parameter

CALVAL Vertical interval

CALVAL instrument

Geophysical parameter

CALVAL Vertical interval

CALVAL instrument

Geophysical parameter

CALVAL Vertical interval

CALVAL instrument

Geophysical parameter

CALVAL Vertical interval

CALVAL instrument

Geophysical parameter

CALVAL Vertical interval

CALVAL instrument

Geophysical parameter

CALVAL Vertical interval

CALVAL instrument

Geophysical parameter

CALVAL Vertical interval

AATSR

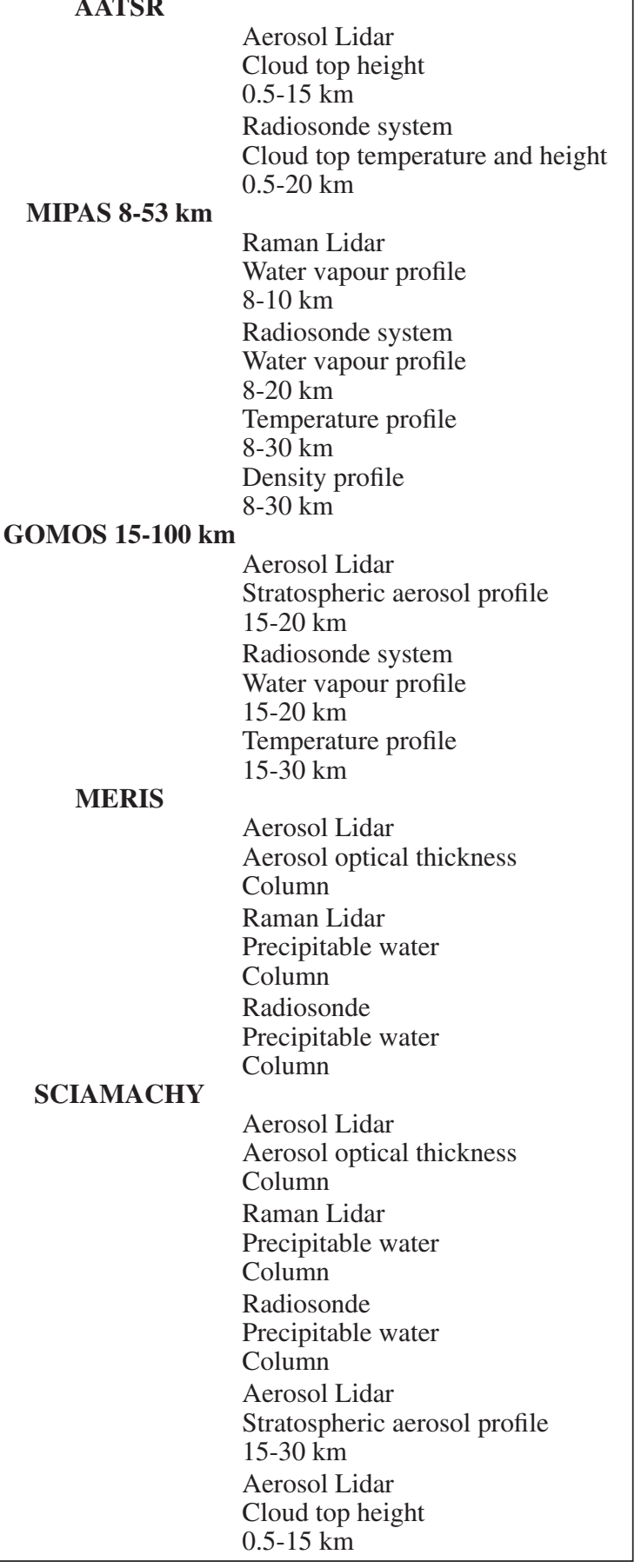


The stratospheric aerosol profile and cloud top height can be retrieved from SCIAMACHY limb measurements and such measurements can be validated by the lidar system. A complete list of validated and validating sensors is given in table I. Calibration and validation measurements (cal/val) will be carried out on a routine basis for the first 6 months after the launch, but will continue afterwards with a reduced schedule for all the duration of the mission.

\section{REFERENCES}

Ambrico, P.F., A. Amodeo, S. Amoruso, M. Armenante, V. BERARDI, A. BosELli, R. BRUZZESE, R. CAPOBIANCO, P. Di Girolamo, L. Fiorani, G. Pappalardo, N. SPINELLI and R. VelotTA (1997): A multiparametric Lidar system spanning from UV to the mid IR, Laser und Optoelektronic, 29, 62-69.

AMBrico, P.F., A. AMODEO, P. Di GIROLAMO and N. SPINELLI (2000): Sensitivity analysis of DIAL measurements in mid IR region, Appl. Opt., 39 (36), 6847-6865.

Bluth, G.J.S., S.D. Doiron, C.C. Schnetzler, A.J. KRUEGER and L.S. WALTER (1992): Global tracking of the $\mathrm{SO}_{2}$ clouds from the June 1991 Mount Pinatubo eruption, Geophys. Res. Lett., 19, 151-154.

CuOMo, V., P. Di Girolamo, F. ESPOSITO, G. PAPPALARDO, C. Serio, N. Spinelli, M. Armenante, B. Bartoli, V. Berardi, R. BRuZzese, C. Bellecci, G.E. CAPUTI, F. De Donato, P. Gaudio and M. Valentini (1997): The LITE correlative measurements campaign in Southern Italy: preliminary results, Appl. Phys. B, B 64 (5), 553-560.

Cuomo, V., P. Di Girolamo, G. Pappalardo, N. SPinelli, M. ARmenAnte, V. BERARdi and M.P. MCCORMicK (1998): Lidar in space technology experiment correlative measurements by lidar in Potenza, Southern Italy, $J$. Geophys. Res., 103, 11,455-11,465.

Di Girolamo, P., R.V. Gagliardi, G. Pappalardo, R.
Velotta, V. BerARdi and N. SPINELli (1995): Twowavelength lidar analysis of stratospheric aerosol size, J. Aerosol Sci., 26 (6), 988-1001.

Di Girolamo, P., G. Pappalardo, V. Berardi, V. CuOmo, N. SPINELLI and R. VELOTTA (1996): Lidar Observations of the stratospheric aerosol layer over Southern Italy in the period 1991-1995, J. Geophys. Res., 101, 18,76518,773 .

Di Girolamo, P., P.F. Ambrico, A. Amodeo, A. Boselli, G. PAppalardo and N. Spinelli (1999): Aerosol observations by lidar in the Nocturnal Boundary Layer, Appl. Opt., 38 (21), 4585-4595.

KASTEN, F. (1968): Falling speed of aerosol particles, J. Appl. Meteorol., 7, 944-947.

McCormick, P.C. and R. VEIGA (1992): SAGE II measurements of early Pinatubo aerosols, J. Geophys. Res., 19. $155-158$.

MCCORMICK, M.P., D.M. Winker, E.V. Browell, J.A. COAKLEY, C.S. GARDNER, R.M. HOFF, G.S. KENT, S.H. Melfi, R.T. MENZIES, C.M.R. Platt, D.A. RANDALl and J.A. REAGAN (1993): Scientific investigations planned for the LIdar in space Technology Experiment (LITE), Bull. Am. Meteorol. Soc., 74, 205-214.

Pappalardo, G., V. CuOMO, N. SPinelli, P. Di Girolamo, V. BERARDI and R. BRUZZESE (1997): Southern Italy correlative Lidar measurements for LITE, in Advances in Atmospheric Remote Sensing with Lidar, edited by A. ANSMANN, R. NEUBER, P. RAIROUX and U. WANDINGER (Springer-Verlag, Berlin), pp. 173.

Pappalardo, G., M. PANDOlFi, P.F. Ambrico, A. Amodeo, P. Di Girolamo and A. Boselli (2001): Lidar measurements of aerosol extinction and backscatter in the PBL, in Advances in Laser Remote Sensing, Selected Papers presented at the 20th International Laser Radar Conference, edited by A. DABAS, C. LOTH and J. PELON, École Polytechnique, 207-210.

POLlaCK, J.B., O.B. TOON, A. Summers, W. VAN CAMP and B. BALDWIN (1976): Estimates of the climatic impact of aerosols produced by space shuttles, SSTs, and other high flying aircraft, J. Appl. Meteorol., 15, 247-258.

ZhaO, J., R.P. TurCo and O.B. ToOn (1995): A model simulation of Pinatubo volcanic aerosols in the stratosphere, J. Geophys. Res., 100, 7315-7328. 